\title{
Listeria Bacteremia: Don't Underestimate Gastroenteritis in Elderly
}

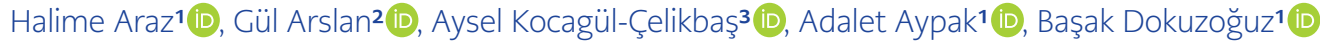 \\ 1 Department of Infectious Diseases and Clinical Microbiology, Ankara City Hospital, Ankara, Turkey \\ 2 Department of Infectious Diseases and Clinical Microbiology, Fatsa State Hospital, Ordu, Turkey \\ 3 Department of Infectious Diseases and Clinical Microbiology, Hitit University School of Medicine, Çorum, Turkey
}

\begin{abstract}
Listeria monocytogenes is a self-limiting gastroenteritis agent in healthy adults, while it can also cause more severe conditions such as bacteremia and meningitis in pregnant women, newborns, elderly and immunosuppressed patients.

In our report, an 81-year-old geriatric patient presented with fever, dysuria, and gastroenteritis. The patient was prescribed ceftriaxone empirically upon admission, and urine culture was reported as Escherichia coli sensitive to ceftriaxone. However, there were still no fever response and clinical improvement on the third day of antibiotic treatment. In blood culture obtained upon admission, Listeria monocytogenes was grown on the third day. The patient responded to treatment after her treatment was changed to ampicillinsulbactam. However, a pulmonary embolism developed on the 14th day of admission, and the patient was transferred to the intensive care unit. In our report, it was stated that attention should be paid to the nutrition of patients in elderly. We also emphasized that blood culture should be taken from every patient who represents with fever and the importance of early and appropriate treatment.
\end{abstract}

Keywords: Listeria monocytogenes, gastroenteritis, bacteremia, geriatric patient

\section{INTRODUCTION}

L isteria monocytogenes is a rare, self-limiting cause of gastroenteritis in healthy adults. It is generally transmitted through foods such as meat, raw vegetables, milk, and dairy products (1). It can cause life-threatening invasive infections in pregnant women, newborns, the elderly, transplant recipients, and patients with an impaired cellular immune response (2-4). In this report, a geriatric case who presented with acute gastroenteritis and was diagnosed as Listeria bacteremia is presented.
Corresponding Author: Halime Araz

E-mail:

halimecavlak@gmail.com

Received: July 05, 2021

Accepted: November 15, 2021

Published: December 30, 2021

\section{Suggested citation:}

Araz H, Arslan G, Kocagül-

Çelikbaş A, Aypak A, Dokuzoğuz B. Listeria bacteremia: don't underestimate gastroenteritis in elderly. Infect Dis Clin Microbiol 2021; 3: 168-172.

DOI: 10.36519/idcm.2021.63 


\section{CASE PRESENTATION}

An 81-year-old female patient was hospitalized with complaints of nausea, vomiting, watery bloodless diarrhea accompanied by tenesmus approximately 20 times a day, dysuria, and pollakiuria for two days. Her anamnesis revealed that she had hypertension, hyperlipidemia and coronary artery disease, she lived with her sister and that her meals were regularly served from a restaurant.

\section{Physical examination}

She was conscious, her fever was $38^{\circ} \mathrm{C}$, her blood pressure was $90 / 70 \mathrm{mmHg}$, and her skin and mucous membranes were dehydrated. Hepatomegaly and suprapubic tenderness were detected.

\section{Laboratory tests}

Leukocytes $12,000 / \mathrm{mm}^{3}$, aspartate aminotransferase (AST) $50 \mathrm{IU} / \mathrm{ml}$, alanine aminotransferase (ALT) $55 \mathrm{IU} / \mathrm{ml}$, urea $20 \mathrm{mg} / \mathrm{dl}$, creatinine $0.8 \mathrm{mg} / \mathrm{dl}$, C-reactive protein (CRP) $187 \mathrm{mg} / \mathrm{L}$ were detected. Plenty of leukocytes were seen in stool microscopy, and any pathogenic bacteria did not grow in the culture. In the complete urinalysis, leukocyte and nitrite positivity were detected. Escherichia coli $10^{3}$ colony-forming unit $(\mathrm{CFU}) / \mathrm{ml}$ sensitive to ceftriaxone grew in urine culture. Stool culture was cultured on blood agar, eosin-methylene blue agar and salmonella-shigella agar as a part of the clinical routine. Pathogenic bacteria did not grow in stool culture. Listeria monocytogenes was grown on the third day in the blood culture of the patient in two vials. Blood samples sent to the laboratory were examined with the BACTEC 9120 (Becton Dickinson, USA) automated blood culture system. Blood cultures that gave positive signals in the device were inoculated on 5\% sheep blood agar, eosinmethylene blue agar and Sabouraud's dextrose agar media. The catalase-positive, oxidase-negative colonies grown on blood media with gram-positive bacillus appearance were loaded into the VITEK® 2 (bioMérieux, Marcy l'Etoile, France) identification device. The strain identified as Listeria monocytogenes by the device was then confirmed by the matrixassisted laser desorption-ionization time-of-flight (MALDI-TOF) mass spectrometry (Bruker Daltonics, Bremen, Germany).

\section{Ultrasonography}

Bilateral grade 1 hydronephrosis and pelvicalyceal ectasia were detected in the kidneys.

\section{Clinical follow-up}

Because during admission, the patient had a fever, leukocytes and nitrite positivity was detected in the complete urinalysis, urinary system infection was considered and intravenous (IV) ceftriaxone treatment was started. Listeria monocytogenes was grown on the third day in the patient's blood culture, whose fever and diarrhea persisted despite appropriate treatment. The treatment was changed to ampicillin-sulbactam $4 \times 2$ gr IV. On the fourth day of ampicillin sulbactam treatment, the patient's fever subsided. On the $14^{\text {th }}$ day of the treatment, a pulmonary embolism developed despite the prophylactic low molecular weight heparin treatment, so she was transferred to the intensive care unit. The patient was followed up in the intensive care unit for six days, and the treatment was completed in three weeks (Figure 1). The patient, whose general condition improved, was placed in a nursing home with the support of the social service.

\section{DISCUSSION}

In geriatric patients, infections may present with different clinical manifestations. Urinary tract infections and urosepsis are the most common infections in this age group. Therefore, if there are urinary symptoms, empirical antibiotic therapy is initiated for the urinary tract. Unnecessary antibiotic treatment in cases with asymptomatic bacteriuria is commonly seen in practice. This may cause the real infectious agent to be missed. Therefore, taking blood cultures from the cases is very important to prevent wrong approaches. A similar condition was experienced in the presented case, and ceftriaxone treatment was started because of urinary symptoms. The presence of ceftriaxone-sensitive Escherichia coli growth in the urine culture also supported the diagnosis. Listeria monocytogenes growth in the blood culture made it possible to recognize the presence of a different infection in the patient.

Listeria monocytogenes is an infectious agent transmitted by consuming contaminated food and can 


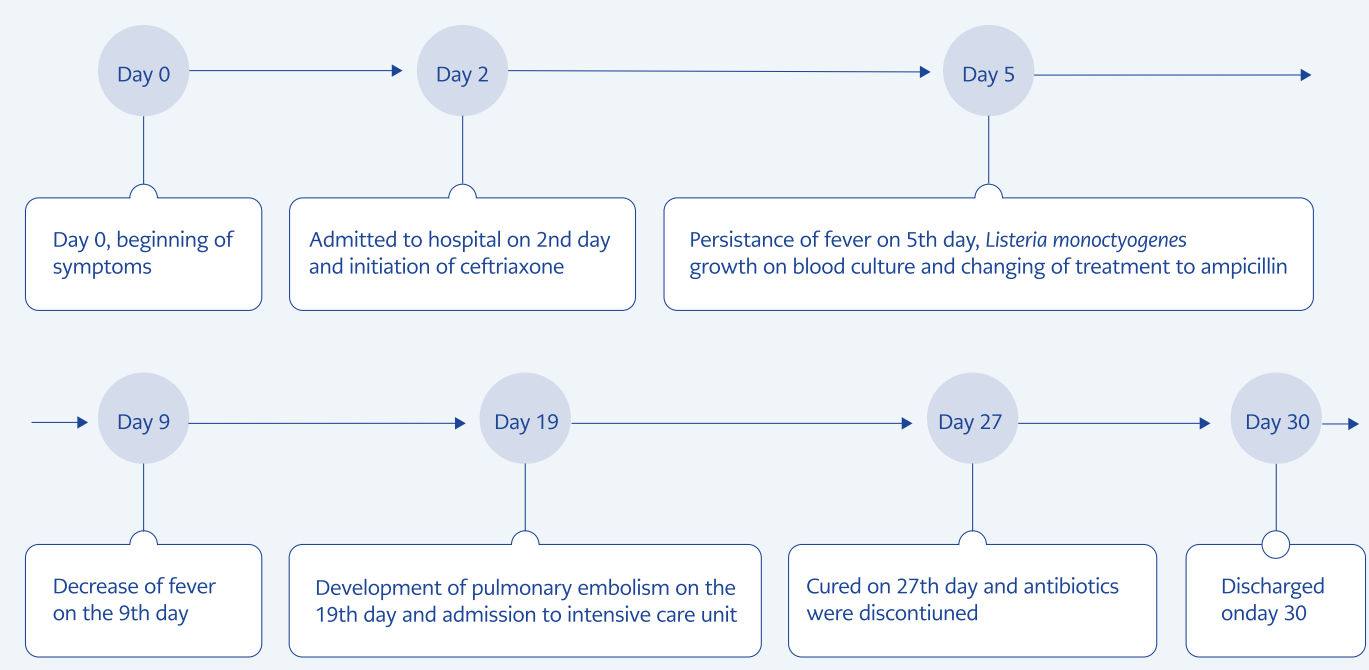

Figure 1. The timeline of the case, summary of the disease, symptoms, treatments, and complications.

cause severe clinical presentations in immunosuppressive individuals, the elderly, and pregnant women (2). The gastrointestinal tract is the most important gateway. Foods made from unpasteurized milk, ready-to-eat, and frozen foods, especially ham, sausage, hot dog, chicken salad, and roast beef have been reported as risky foods (5). Food not prepared in proper conditions, poor storage conditions after preparation, and personnel working in food preparation play an important role in contamination. The fact that the presented case constantly consumed food from a restaurant is a significant risk factor for transmission.

The microorganism often causes central nervous system and bloodstream infections. Listeria monocytogenes does not produce any enterotoxin, and its molecular pathogenesis is not clearly known. It is phagocytized by attaching to the host cell with e-cadherin. It has been shown to cause invasive diarrhea, fever, and bacteremia $(6,7)$. As in our case, acute enteritis may occur either as the sole symptom of Listeria infection or as a finding preceding typical manifestations such as sepsis, meningitis, and encephalitis. A rare complication of Listeria gastroenteritis may lead to severe sepsis and death (2). This condition reveals the importance of taking blood culture in cases presenting with diarrhea, especially in the geriatric age group.
In patients who have diarrhea accompanied by fever, bloody or mucoid stools, severe abdominal cramps or tenderness, or sepsis symptoms, a stool test should be performed for Salmonella spp., Shigella spp., Campylobacter spp., Yersinia spp., Clostridium difficile and Shiga toxin-producing E. Coli (STEC). If enteric fever or bacteremia is suspected, culture-independent diagnostic testing, including panel-based multiplex molecular diagnosis from stool and blood samples, should be performed when indicated. In addition, bone marrow cultures (especially valuable if antimicrobial agents have been administered), stool, duodenal fluid, and urine may be useful in detecting enteric fever (8). However, in our case, only stool sample was cultured on blood agar, eosin-methylene blue agar, and salmonella-shigella agar as a part of the routine, and blood culture was taken for bacteriology because of the fever.

Empirical antimicrobial therapy is recommended in immunocompromised patients with febrile or bloody inflammatory diarrhea (8). In addition, it is recommended that in risk groups for severe listeriosis, such as the elderly, pregnant women, newborns and immunocompromised hosts, gastroenteritis should be treated with ampicillin or trimethoprim-sulfamethoxazole in case of diarrhea.

In a study examining two hundred and twenty-nine Listeria cases, it was reported that cephalosporins 
were used in $90 \%$ of undertreated cases. In the study, it was observed that 195 patients who survived were given adequate and appropriate antibiotic therapy in the early period (9). Similarly, in our case, empirical cephalosporin was started, and no improvement was observed. Mortality of Listeria infection has been reported as $26.6 \%$ and $38 \%$ in different studies $(9,10)$. Early diagnosis and appropriate treatment are lifesaving. Penicillin or ampicillin appears to be the first-choice drug in Listeria treatment (11-13). Depending on the clinical presentation, gentamicin can be added to the treatment. Severe listeriosis infections should be treated with a combination of ampicillin + gentamicin.

Ceftriaxone is not a good option in the treatment of cases (11). In our patient, fever response could be achieved on the seventh day of the disease by changing the treatment to ampicillin sulbactam.
Cases with Listeria bacteremia should be followed closely for the clinical manifestations, including pneumonia, peritonitis, and septic pulmonary embolism, which may be caused by Listeria $(14,15)$, and if possible, severe cases should be followed up under intensive-care conditions.

\section{CONCLUSION}

Special attention should be paid to patients' nutrition in the geriatric age group, unpasteurized dairy products should be avoided, and ready-to-eat food consumption should be limited if possible. In addition, it should be kept in mind that in this age group, infections may present with different clinical manifestations and Listeria may be among the infectious agents, and blood cultures should be taken from all cases with fever.
Ethical Approval: N.A.

Informed Consent: Written consent was obtained from the patient.

Peer-review: Externally peer-reviewed

Author Contributions: Concept - A.K.Ç., B.D.; Design - H.A.; Supervision - A.K.Ç., B.D., A.A.; Fundings - G.A., A.A.; Materials H.A., G.A.; Data Collection and/or Processing - H.A., G.A.; Analysis and/or Interpretation - H.A., A.A.; Literature Review - H.A., G.A.;
Writer - H.A., A.K.Ç.; Critical Reviews - A.K.Ç., B.D.

Conflict of Interest: The authors declare no conflict of interest.

Financial Disclosure: The authors declared that this study has received no financial support.

Scientific presentation: This study was presented as a "poster presentation" at the 19th Turkish Clinical Microbiology and Infectious Diseases Congress on March 28-31, 2021, in Antalya, Turkey.

\section{REFERENCES}

1 Zhu Q, Gooneratne R, Hussain M. Listeria monocytogenes in fresh produce: Outbreaks, prevalence and contamination levels. Foods. 2017;6(3):21. [CrossRef]

2 Mehmood H, Marwat ADJK, Khan NAJ. Invasive Listeria monocytogenes gastroenteritis leading to stupor, bacteremia, fever, and diarrhea: A rare life-threatening condition. J Investig Med High Impact Case Rep. 2017;5(2):2324709617707978. [CrossRef]

3 Tekay F, Özbek E, Kazancı E, Okur N, Demirel M, İpek MŞ. [A Case of Neonatal Sepsis due to Listeria monocytogenes Serotype 4b: Case report and literature review]. Dicle Med J. 2014; 41(3):599-602. Turkish. [CrossRef]

4 Yerlikaya A, Eryılmaz B, Kahyaoğlu B, Ergönül Ö. Rhombencephalitis with Listeria monocytogenes. Infect Dis Clin Microbiol. 2019;1:47-54. [CrossRef]

5 Maurella C, Gallina S, Ru G, Adriano D, Bellio A, Bianchi DM, et al. Outbreak of febrile gastroenteritis caused by Listeria monocytogenes 1/2a in sliced cold beef ham, Italy, May 2016. Euro Surveill. 2018;23(10):17-00155. [CrossRef]
6 Marquis H, Drevets DA, Bronze MS, Kathariou S, Golos TG, Iruretagoyena JI. Pathogenesis of Listeria monocytogenes in Humans. Singh SK, editor. Human Emerging and Re-emerging Infections: Viral and Parasitic Infections, Volume 1. 1st ed.. New Jersey: John Wiley \& Sons, Inc.; 2015. p.749-72.

7 Mengaud J, Ohayon H, Gounon P, Mege RM, Cossart P. E-cadherin is the receptor for internalin, a surface protein required for entry of L. monocytogenes into epithelial cells. Cell. 1996;84(6):923-32. [CrossRef]

8 Shane AL, Mody RK, Crump JA, Tarr PI, Steiner TS, Kotloff K, et al. 2017 Infectious Diseases Society of America Clinical Practice Guidelines for the diagnosis and management of infectious diarrhea. Clin Infect Dis. 2017;65(12):e45-e80. [CrossRef]

9 Thønnings S, Knudsen JD, Schønheyder HC, Søgaard M, Arpi M, Gradel K, et al. Antibiotic treatment and mortality in patients with Listeria monocytogenes meningitis or bacteraemia. Clin Microbiol Infect. 2016;22(8):725-30. [CrossRef] 
10 Zeitlin J, Carvounis CP, Murphy RG, Tortora GT. Graft infection and bacteremia with Listeria monocytogenes in a patient receiving hemodialysis. Arch Intern Med. 1982;142(12):2191-2.

11 Temple ME, Nahata MC. Treatment of listeriosis. Ann Pharmacother. 2000;34(5):656-61. [CrossRef]

12 Evirgen Ö. Listeria monocytogenes infeksiyonu; kliniği, tanı ve tedavi özellikleri. Van Tip Derg. 2005; 12(1):32-5.

13 Pagliano P, Arslan F, Ascione T. Epidemiology and treatment of the commonest form of listeriosis: meningitis and bacteraemia. Infez Med. 2017;25(3):210-6
14 Rajalakshmi A, Gopalakrishnan R, Nambi PS, Rao PV, Ramasubramanian V. Listeria in adults-truly rare or rarely diagnosed in India. J Assoc Phys India. 2017;65:106-8.

15 Tekkanşmaz N, Özelsancak R, Torun D, Alişkan HE. Listeriosis in a patient undergoing hemodialysis: A case report and review of the literature. Turkish J Nephrol. 2017; 26:111-4. [CrossRef] 